\title{
Single-stranded siRNAs for in vivo gene silencing
}

The discovery of RNA interference (RNAi) in the late 1990s was almost immediately recognized as providing a general mechanism for specifically targeting thousands of disease-associated genes. But the difficulty of delivering the necessary RNA oligomers to cells in vivo has vexed the field ever since. Two recent papers in Ce//1,2 now suggest that single-stranded RNAs (ssRNAs) could be a valuable alternative to the more commonly used double-stranded molecules.

Double-stranded, small interfering RNAs (ds-siRNAs) are the most efficient way of inducing RNAi, but they do not readily cross cellular membranes and require special delivery vehicles to gain access to the cytoplasm of cells. The need to formulate ds-siRNAs not only increases the complexity of designing siRNA-based therapies but has also been the source of many of the adverse effects observed in clinical trials of RNA drugs. In addition, both strands of the double-stranded molecule can, in principle, be incorporated into the RNA-induced silencing complexes that mediate RNAi. Although methods to guide the strand selection toward the desired RNA strand have been developed over the years, researchers remain worried about off-target effects and associated toxicities.

In contrast, ssRNAs do not need to be formulated to enter cells and can be delivered in a simple saline solution. By definition, they also eliminate the possibility of off-target effects due to erroneous strand incorporation. Despite these potential advantages, ssRNAs have not been widely used because they are substantially less potent inhibitors of gene expression than their double-stranded counterparts and, even more importantly, are very unstable in serum and in cells.

Lima et al. ${ }^{1}$ and $Y u$ et al. ${ }^{2}$ now show how chemical modifications can be used to increase both the stability and the potency of ss-siRNAs for in vivo applications. The introduction of up to 14 phosphorothioates, alternating 2 '-fluororibose and 2 '-methoxyribose and additional 2 '-methoxyethylribose modifications increases the half-life of the SSRNA in cell homogenates from less than 2 minutes to more than 8 hours. Both papers show that the modified ss-siRNAs engage the cellular RNAi machinery and that the interaction with the key AGO2 protein depends on the presence of a $5^{\prime}$ phosphate. To overcome the rapid dephosphorylation that RNA undergoes in vivo, the authors ${ }^{1,2}$ introduce a metabolically stable 5'-(E)-vinylphosphonate modification. Similarly to siRNAs, ss-siRNAs will lead to degradation

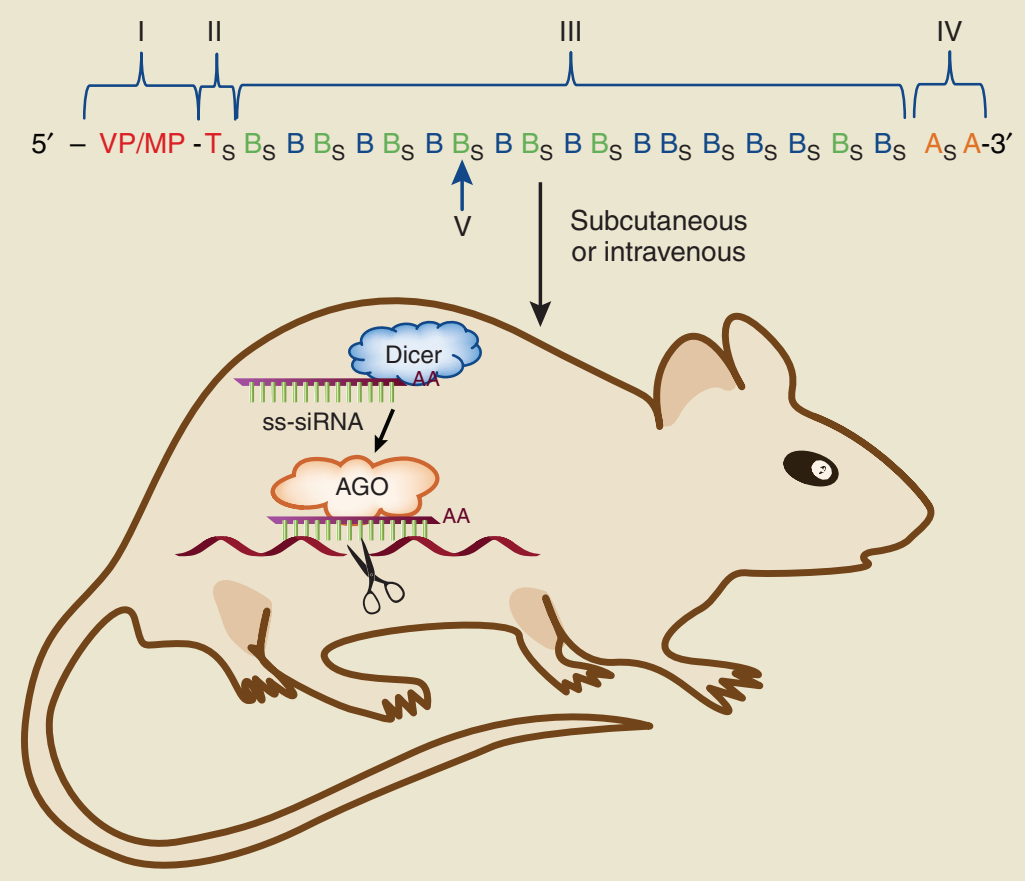

of their target mRNAs when they are perfectly complementary but will only inhibit translation when they contain a central mismatch.

In mice, efficient silencing of the target gene was observed in the liver after either intravenous or subcutaneous delivery ${ }^{1}$ and in the brain after direct intraventricular infusion of the ss-siRNA ${ }^{2}$. In experiments in a Huntington's disease model, Yu et al. ${ }^{2}$ showed that they can design ss-siRNAs that are $>30$-fold more active against the diseasecausing allele of mouse $\mathrm{Htt}$ (huntingtin) than against the normal allele, reducing the abundance of the disease-causing protein by up to $80 \%$. Lima et al. ${ }^{1}$ targeted three different mRNAs (PTEN, Factor VII and ApoC) in wild-type mice, reducing the liver transcript abundance by up to $\sim 70 \%$, especially when an additional lipid modification was introduced that increased targeting to the liver.

Although these papers introduce ss-siRNAs as a potentially useful therapeutic option, it remains to be seen how advantageous these agents will prove to be in practice. Even though the chemically modified ssRNAs are much more effective than the unmodified versions, the doses required for efficient gene silencing are still relatively high. For systemic treatment, doses between $100 \mathrm{mg}$ and $300 \mathrm{mg}$ per kilogram of body weight were needed to achieve a meaningful knockdown of genes in the liver, and only very modest knockdown was observed in other tissues at these doses. Even with direct infusion to the brain, $300 \mu \mathrm{g}$ a day was required. "Toxicity at these doses might be a worry, especially as very little is known about the metabolism of the modified bases. More work is certainly needed," says Phil Zamore of the University of Massachusetts Medical School in Worcester.

"Although the high doses are not necessarily a problem, more follow-up work that carefully characterizes toxicities and especially off-target effects of the ss-siRNA will be needed," says Mark Kay of Stanford University in Palo Alto, California, adding that "one also needs to be careful when extrapolating from mice to man. With the lipid-based delivery vehicles, for example, many problems only became apparent in humans." But as these are the first reports of successful use of ss-siRNAs in vivo, there may be plenty of room for improvement. How rapidly technology can develop has been seen with the ds-siRNA delivery vehicles, in which doses needed for gene knockdown in the liver of mice were brought down from $>10 \mathrm{mg}$ to $<0.01 \mathrm{mg}$ RNA per kilogram of body weight within a few years ${ }^{3}$. "It is still early days for ss-siRNAs," says Kay, "and we don't know what will work best in the end."

\section{Markus Elsner, Associate Editor,} Nature Biotechnology

1. Lima, W.F. et al. Cell 150, 883-894 (2012).

2. Yu, D. Cell 150, 895-908 (2012).

3. Semple, S.C. Nat. Biotechnol. 28, 172-176 (2010). 